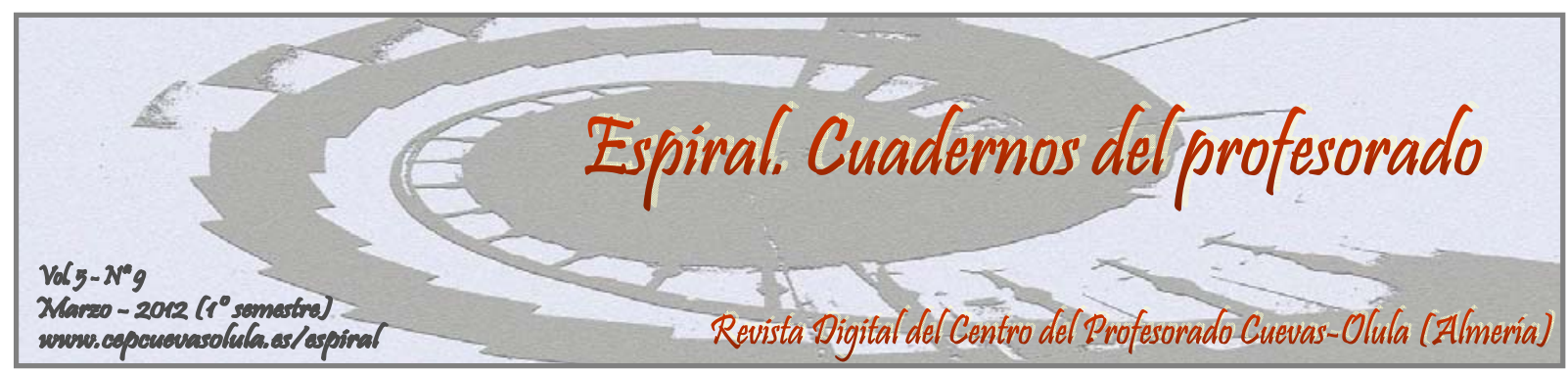

\title{
ANÁLISIS DEL LÉXICO DE APRENDICES DE INGLÉS DE CUARTO DE SECUNDARIA EN E-MAILS
}

\author{
ANALYSIS OF THE VOCABULARY USED IN E-MAILS BY 4TH GRADE \\ SECONDARY EFL LEARNERS
}

\author{
Andrés Canga Alonso
}

\author{
Universidad de La Rioja, Logroño, España
}

\begin{abstract}
RESUMEN: El objetivo principal del estudio es identificar la frecuencia, el número de tipos y ocurrencias y los tipos de palabras utilizados por 44 estudiantes españoles de inglés de $4^{\circ}$ de ESO para describir personas y fiestas tradicionales del Reino Unido a partir de la información obtenida en un intercambio de e-mails con hablantes nativos basado en el e-mail tándem. Los textos producidos por los estudiantes fueron editados en formato electrónico y examinados con los analizadores de vocabulario Frequency y Range para comprobar (i) el número de tipos y ocurrencias, (ii) las clases de palabras empleadas por los alumnos (nombres, adjetivos, verbos y adverbios), y (iii) clasificar los tipos y las ocurrencias de acuerdo con las tres listas de palabras más frecuentes del inglés propuestas por Nation (http://www.victoria.ac.nz/lals/staff/paul-nation.aspx). Los resultados indican que (i) los nombres son las categorías más utilizadas por los estudiantes, seguidos de los verbos; (ii) los nombres pertenecen al campo semántico de la familia y el tiempo libre y (iii) la mayoría de ocurrencias y tipos se encuentran en la lista 1 , si bien más de la mitad de los alumnos emplean al menos un tipo perteneciente a la lista 3.
\end{abstract}

Palabras clave: E-mails, tipos de palabras, frecuencia, ocurrencias y tipos.

ABSTRACT: This study aims at identifying the frequency, number of types and tokens, and the types of words used by 44 Spanish students in their last year of Secondary Education (4 ${ }^{\text {th }}$ ESO) to describe people their same age as well as traditional festivals in the UK by means of an e-mail exchange with native speakers based on e-mail tandem. The texts written by the students were edited electronically and processed with the vocabulary analysing programmes Frequency and Range to test (i) the number of types and tokens, (ii) the types of words used by the students (i.e. nouns, verbs, adjectives and adverbs), and (iii) to classify these tokens and types according to the three lists of most frequently used words in English proposed by Nation (http://www.victoria.ac.nz/lals/staff/paul-nation.aspx). The results show that (i) nouns are the most frequently used types of words, followed by verbs; (ii) nouns belong to the semantic field of family and free time and (iii) most of the tokens and types used are included in list 1 , although more than half of the students are able to use at least one type included in list 3.

Key words: E-mails, types of words, frequency, tokens and types.

Este estudio se ha realizado dentro del marco de un proyecto de investigación financiado por el Ministerio de Ciencia y Tecnología titulado Factores individuales y contextuales en la adquisición y desarrollo de la competencia léxica en inglés como lengua extranjera con número de Referencia: FFI2010-19334 (Subprograma FILO). 
Canga Alonso, A. (2012). Análisis del léxico de aprendices de inglés de cuarto de secundaria en emails. Espiral. Cuadernos del Profesorado, 5(9), 16-25. Disponible en: http://www.cepcuevasolula.es/espiral.

Fecha de recepción: 30/11/2011

Fecha de aceptación: 13/02/2012
Enviar correspondencia a: andres.canga@unirioja.es

\section{1.- INTRODUCCIÓN}

En las últimas décadas, el correo electrónico se ha convertido en uno de los modos de comunicación más frecuentes tanto a nivel personal como profesional por lo que millones de personas de diferentes orígenes socioculturales entran en contacto a través de esta forma de comunicación asíncrona (Danet, 2002). Los adolescentes no han sido ajenos a esta influencia y el e-mail se ha convertido en una de sus herramientas básicas de comunicación interpersonal. Por ello, parece adecuado realizar un estudio sobre el vocabulario utilizado en e-mails por estudiantes de inglés de cuarto de educación secundaria dada la importancia del léxico como factor determinante en la comunicación, y en el éxito o el fracaso del aprendizaje de una lengua extranjera (Nation, 2001). Como se expondrá más adelante, el objetivo principal del presente estudio es trazar el perfil del vocabulario utilizado por aprendices de inglés adolescentes (15-16 años) para describir personas y fiestas tradicionales del Reino Unido a partir de la información obtenida en un intercambio de e-mails llevado a cabo con estudiantes de español de su misma edad de un centro educativo del Reino Unido.

$\mathrm{Al}$ contrario que la carta personal que presenta rasgos típicos de la comunicación escrita entre los que se incluyen: brevedad, estilo apropiado, organización adecuada, conclusiones finales y uso de rituales como saludos y despedidas (Jiménez Catalán y Ojeda Alba, 2007b, p. 506), el e-mail combina elementos propios del lenguaje escrito y de la lengua oral (Biesenbach-Lucas, 2007; BiesenbachLucas y Weasonforth, 2001; Crystal, 2001; Chi-Fen, 2006; Danet, 2002).

El uso del e-mail como herramienta pedagógica en el aula de idiomas ha sido debatido por los expertos en didáctica de lenguas desde mediados de los años 90 hasta la actualidad. En esta línea, Belisle (1996) pone de manifiesto las ventajas que se derivan del uso del e-mail en el aula, puesto que permite a los estudiantes entrar en contacto directo entre sí y con el profesor, al tiempo que les permite familiarizarse con una herramienta básica del siglo XXI. El e-mail fomenta la colaboración entre estudiantes con diferentes lenguas maternas, favoreciendo el desarrollo de las destrezas de expresión escrita y acerca la realidad de la lengua extranjera al aprendiz ya que entra en contacto directo con hablantes de la lengua que se propone aprender (Little, 1998).

Esta forma de comunicación asíncrona se convierte, por tanto, en el texto auténtico, entendido como interacción auténtica entre individuos con diferentes lenguas maternas lo que conlleva un mayor grado de implicación por parte del aprendiz que el que se produce en la clase de lenguas tradicional. La mayor implicación del alumnado en las actividades educativas lleva implícito un grado de motivación hacia su aprendizaje cuando pueden utilizar el e-mail en sus interacciones cotidianas (Nagel, 1999).

Por otro lado, la colaboración con individuos pertenecientes a una comunidad lingüística distinta a la propia ayuda a admitir las debilidades y las fortalezas que posee un determinado aprendiz para ir superándolas o afianzándolas con la ayuda del compañero. Para Gonglewski, Meloni y Brant (2001), el uso del e-mail permite ampliar el espacio y el tiempo de aprendizaje de la lengua meta, puesto que los discentes no necesitan estar en el aula a una hora determinada para establecer contacto con hablantes de la lengua meta, sino que pueden conectarse desde su casa, la biblioteca o cualquier otro lugar que tenga acceso a Internet, ampliándose, de este modo, el lugar desde donde entran en contacto con sus interlocutores y el tiempo que pueden dedicar a la redacción y lectura de los textos que escriban en la lengua meta, puesto que no estarán limitados por el tiempo de duración de la clase. 
Por último, el e-mail les proporciona un contexto adicional para la discusión que está relacionado con los temas abordados en el aula (Gonglewski et al., 2001), con el objetivo favorecer la adquisición de vocabulario relacionado con el tema propuesto en la lengua meta.

Teniendo en cuenta estas premisas, en el año académico 1993-1994, gracias al programa Lingua subvencionado por la Unión Europea, se crea el International E-mail Tandem Network (Brammerts y Little, 1996; Álvarez, Blanco, Ojanguren, Brammerts y Little, 1996). Mediante dicho programa se estableció una red de comunicación en tándem entre instituciones universitarias de más de diez países europeos. El e-mail tándem permite a escolares, estudiantes universitarios o profesionales de cualquier campo establecer contacto con individuos que son hablantes nativos de la lengua que quieren aprender y que, a su vez, están interesados en mejorar su competencia comunicativa en la lengua materna de su compañero tándem, aprender sobre aspectos relacionados con la cultura meta y beneficiarse de sus conocimientos y experiencia (Appel, 1999; Brammerts, 2003; Vinagre, 2005).

Por medio del e-mail tándem se establece una comunicación asíncrona basada en la comunicación escrita (Brammerts y Calvert, 2003). Este tipo de comunicación posee una serie de ventajas para los alumnos, puesto que los mensajes se encuentran accesibles para los aprendices y pueden ser releídos y utilizados con distintos fines tantas veces como se considere necesario. Además, los estudiantes tienen más tiempo para elaborar sus textos y pueden recurrir a otras fuentes de información siempre que lo estimen necesario y conveniente.

Esta metodología se sustenta en dos principios: reciprocidad y autonomía. El principio de reciprocidad implica aprender del compañero, ya que ambos miembros de la pareja tienen como objetivo aprender la lengua materna del otro miembro de la pareja tándem. Para ello, cada uno de ellos aporta los conocimientos de su lengua y cultura que, a su vez, son los que su compañero desea adquirir, así como el compromiso de ayudarse mutuamente en su proceso de aprendizaje. Con el objetivo de extraer el mayor beneficio de la experiencia, se deben utilizar la lengua materna y la lengua meta en cada e-mail, siendo la situación ideal cuando el alumno escribe la mitad del mensaje en cada una de las lenguas. Esta situación le lleva a reflexionar sobre su lengua materna para responder a las demandas de su compañero y también sobre la lengua meta cuando se expresa en la lengua extranjera para mostrar sus inquietudes, dudas o deseos de adquirir nuevos conocimientos sobre el entorno sociocultural del país extranjero.

El principio de autonomía permite a los discentes responsabilizarse de su propio proceso de aprendizaje. La toma continua de decisiones en el proceso de aprendizaje implica que los discentes deben adaptar sus necesidades a las potencialidades, limitaciones e intereses del compañero y establecer las pautas de su comunicación en tándem. Por otro lado, es posible que los aprendices tengan diferentes objetivos en su instrucción siendo la negociación entre los miembros de la pareja un elemento clave en el éxito de su aprendizaje.

En lo que respecta a los objetivos didácticos, se podrían resumir en tres: capacidad para comunicarse en la lengua meta que es la lengua nativa del compañero (comunicación intercultural), capacidad para desenvolverse de forma apropiada en un contexto cultural distinto al materno y capacidad para colaborar con el compañero con el objeto de lograr las metas propuestas (Brammerts, 2003).

Los aprendices que se propongan alcanzar estos objetivos han de tener siempre al compañero como modelo, puesto que el input léxico y socio-cultural que éste le proporcione a través del e-mail que le envíe, será el texto que tomará como base principal de su aprendizaje. Asimismo, este modelo de aprendizaje favorece la adquisición de vocabulario relacionado con los temas tratados en cada email tal como se pone de manifiesto en los resultados obtenidos por los participantes en el presente estudio y que se explicarán en detalle en el tercer epígrafe de este artículo.

\section{OBJETIVOS}

El objetivo del estudio es trazar el perfil del vocabulario utilizado por aprendices de inglés adolescentes (15-16 años) a la hora de describir personas y fiestas tradicionales del Reino Unido 
tomando como referencia la información obtenida en el intercambio de e-mails basado en el e-mail tándem llevado a cabo con estudiantes de español de su misma edad de un centro educativo del Reino Unido. Asimismo, a partir del análisis de estos textos, se pretende identificar: (i) el número de tipos y ocurrencias, (ii) las clases de palabras empleadas por los alumnos (nombres, adjetivos, verbos y adverbios) (Greenbaum y Quirk, 1993), asi como (iii) clasificar los tipos y las ocurrencias de acuerdo con las tres listas propuestas por Nation (http://www.victoria.ac.nz/lals/staff/paul-nation.aspx). La lista 1 incluye las 1000 palabras más frecuentes del inglés. En la lista 2 se encuentran las 2000 más frecuentas y la lista 3 recoge las palabras que están por encima de las listas 1 y 2 que son utilizadas con frecuencia por estudiantes de secundaria y primeros niveles postobligatorios.

\section{3.- MÉTODO}

\section{Participantes, procedimientos e instrumentos}

El presente estudio se basa en un corpus de 44 e-mails escritos por 44 aprendices de inglés en $4^{\circ}$ ESO. La muestra de informantes proviene de un centro de secundaria ubicado en el Principado de Asturias. Dicha muestra es homogénea en lo que respecta a edad y extracto socio-cultural. Los informantes tienen entre 15 y 16 años, comparten una misma lengua materna y provienen de clases medias. En el momento de recogida de datos, los estudiantes han tenido más de 600 horas de exposición al inglés mediante las clases de instrucción regular. Con el objeto de conseguir un centro con el que poder llevar a cabo la experiencia, se recurrió a la Agencia Tándem: (tandem@slf.ruhr-uni-bochum.de) quien de acuerdo con las características de los alumnos, el grupo de edad al que pertenecen y la similitud en cuanto a sus objetivos didácticos, asoció al centro educativo español con un colegio situado en West Sussex (Inglaterra) donde se estudia español como segunda lengua extranjera.

Los alumnos fueron distribuidos en parejas intentando que el grado de conocimiento de la lengua extranjera fuese similar en ambos miembros para evitar que se produjesen problemas de comunicación y la experiencia resultase productiva para ambos aprendices (Gläsmann y Calvert, 2001; Brammerts y Kleppin, 2003).

Antes de comenzar con el trabajo propiamente dicho, se dedica una sesión para explicar la dinámica de trabajo que se llevará a cabo a lo largo del curso, dejando claro la importancia de la colaboración mutua entre los miembros de la pareja para poder realizar las diferentes tareas. El intercambio de e-mails se realiza dos veces al mes en el aula de informática. Los alumnos redactan su e-mail y lo almacenan en la carpeta habilitada al efecto en la intranet del centro. Una vez se ha completado la tarea, el profesor se encarga de remitirlas a su colega que, a su vez, las distribuye a sus alumnos. El docente envía el mensaje tal como lo recibe, puesto que es el compañero tándem el encargado de corregir los errores léxicos y gramaticales que pudiera contener, siguiendo las pautas explicadas por el profesor al comienzo del trabajo en tándem. Con este sistema se pretende que todos los participantes reciban regularmente los mensajes de su pareja y que los alumnos respondan a las tareas que se les plantean, evitando, así, que caigan en la tentación de tratar otros temas que no estén relacionados con lo propuesto en la actividad.

Para la elección de los temas se tuvieron en cuenta las propuestas de Brammerts y Calvert (2003) aplicadas al tándem presencial que fueron adaptadas al e-mail tándem por Gläsmann y Calvert (2001). Brammerts y Calvert defienden que las actividades deben estimular la comunicación auténtica entre los miembros de la pareja. Por ello, han de ser lo suficientemente abiertas para que cada alumno pueda incluir experiencias, ideas u opiniones partiendo de sus conocimientos previos. Asimismo, las tareas tienen la misión de motivar a los participantes a continuar con su trabajo en tándem. Considerando estas tres razones, los autores mencionados sugieren cinco tipos de actividades aplicables al tándem presencial: "Sharing personal information; exchanging information; eliciting and discussing points of view; being creative together, and talking about language and communication" (Brammerts y Calvert, 2003, p. 58-59).

El primer tipo de actividades tiene como objetivo despertar la curiosidad en cada uno de los miembros de la pareja en lo que respecta a su personalidad, gustos y aficiones. Este tipo de actividad 
se refleja en los dos primeros mensajes que elaboran los alumnos una vez conocen la identidad de sus parejas.

En lo que respecta a las tareas de intercambio de información cada miembro de la pareja se convierte en la fuente de información que el compañero debe tomar como referencia para poder realizar la tarea encomendada. Este tipo de tareas constituye el núcleo del trabajo realizado por los discentes durante el curso escolar, en el que se abordaron las siguientes tareas:

Teniendo en cuenta las características y principios del e-mail tándem expuestos en la introducción de este artículo, los objetivos específicos de la investigación apuntados en el Tabla 1.- Tareas.

Mensaje de introducción

Descripción personal: gustos, aficiones y personalidad

Fiestas en España e Inglaterra

Navidad

Nuestro Colegio

Vacaciones apartado 2 (número de ocurrencias y tipos utilizados por cada informante, los tipos de palabras empleadas y su clasificación en las tres listas de frecuencia propuestas por Nation) y la temática abordada durante los tres primeros meses del curso (descripción de personas, gustos y aficiones fiestas tradicionales en Inglaterra en concreto Halloween y Guy Fawkes' Night), se pidió a los estudiantes que elaborasen un e-mail que debía ser remitido al profesor. Dicho e-mail debía responder al siguiente anunciado: Describe your e-mail tándem and talk about festivals in England. El objetivo de la tarea era comprobar si los participantes eran capaces de proporcionar información referente tanto a la descripción personal de sus compañeros tándem como sobre las fiestas en Inglaterra. El texto a remitir debía tener una extensión mínima de 6 líneas. Cinco estudiantes enviaron textos de una extensión inferior a la solicitada por lo que debieron ser excluidos de la muestra, quedando éste reducida a 39 emails.

Todos los textos producidos por los estudiantes fueron editados en formato electrónico y sometidos a revisión ortográfica para evitar un recuento ficticio de ocurrencias y tipos que no se encuentren recogidos en los diccionarios de inglés contemporáneo al uso. Posteriormente, fueron sometidos al análisis mediante los analizadores de vocabulario Frequency y Range (http://www.victoria.ac.nz/lals/staff/paul-nation.aspx) para analizar el número de tipos y ocurrencias relacionados con la descripción de personas, gustos, aficiones y las fiestas en el Reino Unido utilizadas por los alumnos en sus e-mails. Partiendo de las definiciones de tipo y ocurrencia (Nation, 2001) se considera ocurrencia el número total de palabras utilizadas en cada e-mail, mientras que tipo se refiere al número de palabras distintas empleadas por cada estudiante en los textos analizados.

Tomando como referencia el análisis de los resultados aportados por Frequency, se elaboran listas de palabras en función de su categoría (Greenbaum y Quirk, 2003) y, de este modo, calcular el porcentaje de nombres, adjetivos y adverbios que se emplean en los e-mails analizados. Esta clasificación permite establecer comparación con estudios previos sobre vocabulario en Educación Primaria en la Comunidad Autónoma de La Rioja en los que se emplea como instrumento de evaluación una carta que los estudiantes debían escribir a una familia inglesa imaginaria con la que iban a convivir durante un periodo de tiempo (Agustín Llach y Barreras Gómez, 2007; Jiménez Catalán y Ojeda Alba, 2007a y 2008; Ojeda Alba, 2010) usando Wordsmith Tools ${ }^{1}$ como herramienta de análisis de vocabulario producido por sus estudiantes. A pesar de la aparente diferencia entre ambos grupos de alumnos por razones de edad y exposición a la lengua extranjera, es posible comparar ambos grupos puesto que no existe una legislación educativa que indique el número de tipos y ocurrencias que deben dominar los alumnos en cada nivel educativo lo que implica que los libros de texto puedan utilizar diferente número de palabras, tipo de vocabulario en el mismo nivel educativo y se repita vocabulario referente a los mismos campos semánticos en ambos niveles educativos (Jiménez Catalán y Mancebo Francisco, 2008) lo que podría implicar que no existiesen diferencias entre los informantes de primaria y secundaria. Por otro lado, el origen sociocultural del alumnado participante

\footnotetext{
${ }^{1}$ Para más información sobre esta herramienta de análisis de vocabulario consultar: http://www.lexterm.unb.br/documentos/WSHELL.pdf. [Consulta: 2011, 30 de noviembre].
} 
y la naturaleza monolingüe del Principado de Asturias y La Rioja hacen que se pueda establecer otra similitud entre los grupos.

Por último, los tipos y las ocurrencias utilizadas por los informantes se clasifican de acuerdo con su pertenencia a las tres listas propuestas por Nation (http://www.victoria.ac.nz/lals/staff/paulnation.aspx) que se corresponden con las 1000 (lista 1), 2000 (lista 2) y palabras que se están por encima de las 2000 palabras más frecuentes del inglés y que son utilizadas con frecuencia por estudiantes de secundaria y primeros niveles postobligatorios (lista 3).

\section{4.- RESULTADOS}

Los datos de los 39 e-mails analizados muestran que los informantes producen un número total de 3105 ocurrencias y 2086 tipos lo que hace que la media de ocurrencias por cada texto sea de 79,62 mientras que la de tipos es de 53,48.

En lo que respecta a la distribución del vocabulario utilizado por los alumnos en función de la categoría del término empleado: nombres, verbos, adverbios y adjetivos, se constata que los nombres constituyen las ocurrencias más utilizadas por los alumnos, seguido de verbos, adjetivos y adverbios.

Tabla 2.- Tipos de palabras.

\begin{tabular}{cc}
\hline CATEGORÍA & PORCENTAJE \\
\hline Nombres & $56 \%$ \\
Verbos & $32 \%$ \\
Adjetivos & $2 \%$ \\
Adverbios & $10 \%$ \\
\hline
\end{tabular}

Estos datos guardan similitud con los obtenidos por aprendices de inglés de primaria en composiciones escritas (Agustín Llach y Barreras Gómez, 2007; Jiménez Catalán y Ojeda Alba, 2007a y 2008; Ojeda Alba, 2010), puesto que los nombres son también la categoría más empleada por los estudiantes de primaria en sus composiciones.

Tal como se pone de manifiesto en la tabla 3, en la que se reflejan las ocurrencias utilizadas con mayor frecuencia por los estudiantes en sus e-mails empleando la herramienta Frequency, se observa que Halloween es el término con mayor número de apariciones (47) ${ }^{2}$ seguido de people (35). Guy (27) y Fawkes (26) aparecen inmediatamente después lo que pone de manifiesto el uso de ambos términos por parte del alumnado para referirse a las fiestas en el Reino Unido a partir de la información recabada en los e-mails enviados por sus parejas tándem. Por ello, el término England presenta una frecuencia de 28 y la palabra festival de 14. Los vocablos sweets (17), houses (15) trick (14), treat (14) y October (12) indican que un porcentaje cercano al $40 \%$ de los estudiantes han asimilado los conceptos básicos directamente relacionados con la noche de Halloween si bien no están muy familiarizados con el concepto pumpkin ya que únicamente se menciona en dos los treinta y nueve mensajes analizados.

En lo que respecta a Guy Fawkes’ Night parece que los alumnos conocen la fecha celebración de este evento en el Reino Unido, puesto que la palabra November aparece en 15 ocasiones en sus narraciones. Sin embargo, no parecen ser muy conscientes del modo en que se celebra dicho evento ya que fireworks únicamente aparece en ocho ocasiones y las alusiones a otros términos relacionados con el evento son muy escasas (por ejemplo, Bonfire (1)).

En cuanto a las descripciones personales, gustos y aficiones, es destacable el escaso número de adjetivos empleados por los participantes para describir a sus parejas tándem, ya que únicamente se constata la presencia del vocablo old con 29 apariciones combinadas con el nombre years para indicar la edad del compañero inglés siendo muy escasas las descripciones físicas con cuatro referencias al pelo (brown hair) y tres a los ojos (blue eyes). Por este motivo, se deduce que los estudiantes se han centrado en otro tipo de rasgos para describir a sus parejas tándem. En primer lugar, indican su nombre lo que hace que el término name presente una frecuencia de 27, para, posteriormente, referirse a términos relacionados con la familia (brother (14), family (14), father (11), mother (9), sister (9)) y

\footnotetext{
${ }^{2}$ En adelante se hará constar entre paréntesis el número que indica la frecuencia de los términos que sean empleados para ejemplificar las palabras utilizadas por los alumnos en sus e-mails.
} 
en tercer lugar se refieren a sus gustos y aficiones empleando palabras como friends (11) o football (11).

Estos resultados se asemejan a los obtenidos por los alumnos de los últimos cursos de educación primaria en La Rioja (Agustín Llach y Barreras Gómez, 2007; Jiménez Catalán y Ojeda Alba, 2007a y 2008; Ojeda Alba, 2010), con lo que parece que no se aprecian diferencias ostensibles entre ambos grupos de alumnos a pesar de que los estudiantes que participan en el estudio objeto de esta investigación han tenido mayor exposición a la lengua inglesa. Esto se puede deber al hecho de que en plena adolescencia los alumnos dan mucha importancia a sus relaciones de amistad y también a la carencia de una legislación educativa que indique el número de tipos y ocurrencias que deben dominar los alumnos en cada nivel educativo de modo que los libros de texto puedan fuesen más homogéneos en cuanto al número de palabras que se incluyen en cada nivel y el tipo de vocabulario que los alumnos deben aprender (Jiménez Catalán y Mancebo Francisco, 2008).

Los verbos empleados por los alumnos participantes en este estudio (ver tabla 4) están directamente relacionados con la temática trabajada en el intercambio de e-mails con sus compañeros ingleses siendo el verbo to be el más usado en su forma del singular is (160) seguido de verbos que indican posesión (has [31]), gustos o preferencias (likes [29], like [10]) y lugar de residencia (lives [27]). Estrechamente ligado a la descripción de gust formas go (20), going (16), goes (14) y went (10) para expresar los lugares donde acuden sus compañeros en su tiempo libre. Así mismo, es de resaltar el uso de verbo celebrate en sus formas de

Tabla 4.- Verbos más frecuentes en los e-mails.

\begin{tabular}{llll}
\hline Palabra & Frecuencia & $\%$ & \% acumulado \\
\hline IS & 160 & 5.2 & 10.58 \\
HAS & 31 & 1.01 & 38.22 \\
LIKES & 29 & 0.94 & 40.17 \\
LIVES & 27 & 0.88 & 44.73 \\
GO & 20 & 0.65 & 50.72 \\
GOT & 20 & 0.65 & 51.37 \\
ARE & 17 & 0.55 & 53.16 \\
GOING & 16 & 0.52 & 54.79 \\
CELEBRATE & 15 & 0.49 & 55.79 \\
CELEBRATED & 15 & 0.49 & 56.28 \\
GOES & 14 & 0.46 & 61.00 \\
CALLED & 13 & 0.42 & 63.70 \\
PLAYING & 12 & 0.39 & 66.93 \\
LIKE & 10 & 0.33 & 69.73 \\
WENT & 10 & 0.33 & 70.70 \\
DO & 9 & 0.29 & 71.61 \\
\hline
\end{tabular}

Tabla 3.- Nombres más frecuentes en los e-mails.

\begin{tabular}{lccc}
\hline \multicolumn{1}{c}{ Palabra } & Frecuencia & $\%$ & \% acumulado \\
\hline HALLOWEEN & 47 & 1.53 & 34.99 \\
PEOPLE & 35 & 1.14 & 36.13 \\
YEARS & 29 & 0.94 & 42.06 \\
ENGLAND & 28 & 0.91 & 42.97 \\
GUY & 27 & 0.88 & 43.85 \\
NAME & 27 & 0.88 & 45.61 \\
FAWKES & 26 & 0.85 & 46.45 \\
SWEETS & 17 & 0.55 & 54.26 \\
HOUSES & 15 & 0.49 & 57.26 \\
NOVEMBER & 15 & 0.49 & 58.24 \\
BROTHER & 14 & 0.46 & 59.18 \\
DAY & 14 & 0.46 & 59.64 \\
FAMILY & 14 & 0.46 & 60.09 \\
FESTIVAL & 14 & 0.46 & 60.55 \\
TREAT & 14 & 0.46 & 62.37 \\
TRICK & 14 & 0.46 & 62.83 \\
CHILDREN & 12 & 0.39 & 65.36 \\
NIGHT & 12 & 0.39 & 65.76 \\
OCTOBER & 12 & 0.39 & 66.15 \\
FATHER & 11 & 0.36 & 67.64 \\
FOOTBALL & 11 & 0.36 & 68.00 \\
FRIENDS & 11 & 0.36 & 68.72 \\
PARTNER & 11 & 0.36 & 69.08 \\
E-TANDEM & 10 & 0.33 & 69.40 \\
CHRISTMAS & 9 & 0.29 & 71.32 \\
MOTHER & 9 & 0.29 & 71.91 \\
SISTER & 9 & 0.29 & 72.20 \\
FIREWORKS & 8 & 0.26 & 73.01 \\
\hline
\end{tabular}
presente y pasado celebrate (15), celebrated (15) para referirse a las fiestas tradicionales abordadas en el intercambio de e-mails.

Los adverbios, al igual que ocurría con los adjetivos, son escasamente empleados por los participantes en este estudio. Así, entre las 50 palabras más frecuentes únicamente encontramos cinco adverbios (10\%): very (13) there (10), when (10), lot (10) y also (8), aspecto éste que concuerda con el escaso número de adjetivos presentes en la muestra puesto que los adverbios suelen modificar a adjetivos, salvo en el caso de los adverbios de lugar y tiempo (Greenbaum y Quirk, 2003).

Tras examinar los e-mails con el programa Range, todos los alumnos utilizan algún término incluido de la lista 1, el 94,7\% emplea al menos una palabra perteneciente a la lista 2 y el índice 
porcentual desciende hasta el $51,28 \%$ en lo que respecta a la lista tres.

Si se analiza el número de ocurrencias y tipos que aparecen en los e-mails de acuerdo con las tres listas mencionadas, se observa (tabla 5) que un 82,8\% de las ocurrencias pertenecen a la lista 1 , descendiendo progresivamente el índice porcentual en las palabras incluidas dentro de las listas 2 y 3 con un $6,5 \%$ y un $1 \%$, respectivamente. En cuanto a los tipos se aprecia un descenso en los vocablos correspondiente a la lista 1 (77,6\%), mientras que hay un incremento en las listas dos y tres alcanzando un $8,4 \%$ y $1,5 \%$, respectivamente.

Estos resultados, al igual que ocurría al analizar la categoría a la que pertenecen la mayor parte de las palabras empleadas por los alumnos, guardan similitud con los obtenidos por (Agustín Llach y Barreras Gómez, 2007; Jiménez Catalán y Ojeda

Tabla 5.- Porcentaje de ocurrencias y tipos aplicando Range.

\begin{tabular}{ccc}
\hline Lista & Ocurrencia & Tipo \\
\hline 1 & $82.8 \%$ & $77.6 \%$ \\
2 & $6.5 \%$ & $8.4 \%$ \\
3 & $1 \%$ & $1.5 \%$ \\
\hline
\end{tabular}
Alba, 2007a y 2008; Ojeda Alba 2010) puesto que el mayor número de tipos y ocurrencias se encuentran en la banda de las 1000 más frecuentes (lista 1), si bien el número de ocurrencias/tipos correspondientes a los niveles dos y tres es ligeramente superior en los alumnos que tomaron parte en el estudio basado en el e-mail tándem.

\section{5.- CONCLUSIONES}

Los resultados del presente estudio ponen de manifiesto que los informantes emplean mayoritariamente nombres pertenecientes a los campos semánticos de algunas fiestas tradicionales del Rerino Unido (Halloween y Guy Fawkes’ Day), la familia, los gustos y las aficiones.

Los verbos constituyen el segundo tipo de palabras más utilizadas por los alumnos y están directamente relacionados con los nombres mencionados anteriormente, puesto que la mayoría de ellos denotan movimiento, gustos, preferencias y posesión. Estos datos parecen indicar que las tareas basadas en el e-mail tándem han ayudado a los alumnos a adquirir el vocabulario relacionado con los temas propuestos a principios de curso. Sin embargo, resulta destacable, el escaso número de adjetivos y adverbios empleados en las descripciones del compañero. Esta situación puede deberse a la naturaleza del e-mail que como se mencionó en la introducción combina rasgos del lenguaje escrito y la comunicación oral (Crystal 2001; Biesenbach-Lucas y Weasonforth 2001; Danet 2002; Chi-Fen 2006; Biesenbach 2007) siendo ésta mucho más directa en contextos informales como es el caso de un intercambio de e-mails entre personas de la misma edad, tendiendo así a evitar el uso de un lenguaje más formal en el que se incluyan mayor número de descripciones y, por tanto, de adjetivos y adverbios que los modifiquen.

Por otra parte, las ocurrencias y los tipos más utilizados por los informantes en sus e-mails se encuentran, en su mayoría, dentro de la lista 1 (1000 palabras más frecuentes del inglés), aspecto éste que coincide nuevamente con los resultados obtenidos por los alumnos y alumnas de Educación Primaria, con lo que parece haber tenido una mayor exposición a la lengua no implica un mayor grado de competencia léxica en la lengua extranjera, si bien el porcentaje de uso de términos incluidos en la lista 2 (2000 palabras más frecuentes del inglés) es superior en el grupo de estudiantes analizados en el presente estudio. Este situación podría deberse a la falta de unas directrices que indiquen el número de palabras que los alumnos deben dominar al final de cada etapa educativa y a la temática de los libros de texto que, en algunos casos, es repetitiva lo que no favorece la adquisición de nuevo vocabulario (Jiménez Catalán y Mancebo Francisco, 2008).

Dada la carencia de investigaciones como la que se presenta en este estudio a nivel de Enseñanza Secundaria Obligatoria parece oportuno continuar en esta línea de investigación y hacer estudios comparativos entre informantes de diferente capacidad del último curso del citado nivel educativo que trabajen con un modelo basado en el e-mail tándem para contrastar los resultados y analizar en el vocabulario que emplean ambos grupos el número de ocurrencias y tipos, la categoría a la que pertenecen dichos términos y su frecuencia de uso para constatar si existen diferencias significativas entre ambos grupos o si las dificultades de aprendizaje no son un obstáculo insalvable 
para la adquisición del vocabulario en la lengua extranjera. Por otro lado, se podrían detallar las ocurrencias y los tipos pertenecientes a los listas 2 y 3 para analizar los clases de palabras de las que se trata y su relación con la temática abordada en el intercambio de e-mails y su relación con los libros de texto utilizados en la instrucción en lengua inglesa. Por último, se podría analizar los resultados obtenidos por los chicos y las chicas en ambos grupos estableciendo comparaciones entre grupos y sexos.

\section{6.- REFERENCIAS}

Agustín Llach, M.P. \& Barreras Gómez, A. (2007). Vocabulary acquisition and use in the written production of young Spanish EFL learners. RESLA, 20, 9-20.

Alvarez, J.A., Blanco, M., Ojanguren, A., Brammerts, H. y Little, D. (1996). Guía para el aprendizaje de lenguas en tándem a través de Internet. Oviedo: Servicio de Publicaciones, Universidad de Oviedo.

Appel, M.Ch. (1999). Tandem language learning by e-mail: Some basic principles and a case study. CLCS Ocassional Paper, 54, 1-64.

Belisle, R. (1996). E-mail activities in the ESL writing class. The Internet TESL Journal, II(12). Disponible en: http:// http://iteslj.org/Articles/Belisle-Email.html. [Consulta: 2011, 29 de noviembre].

Biesenbach-Lucas, S. (2007). Students writing emails to faculty: an examination of e-politeness among native and non-native speakers of English. Language Learning \& Technology, 11(2), 59-81.

Biesenbach-Lucas, S. y Weasenforth, D. (2001). E-mail and word processing in the ESL classroom: how the medium affects the message. Language Learning \& Technology, 5(1), 135-165.

Brammerts, H. (2003). Autonomous language learning in tandem: The development of a concept. En T. Lewis \& L. Walker (Comps.), Autonomous language learning in tandem (pp. 27-36). Sheffield: Academy Electronic Publications.

Brammerts, H. \& Calvert, M. (2003). Learning by communicating in tandem. En T. Lewis \& L. Walker (Comps.), Autonomous language learning in tandem (pp. 45-59). Sheffield: Academy Electronic Publications.

Brammerts, H. \& Kleppin, K. (2003). Supporting face-to-face tandem learning. En T. Lewis \& L. Walker (Comps.), Autonomous language learning in tandem (pp. 157-168). Sheffield: Academy Electronic Publications.

Brammerts, H. \& Little, D. (Comps.), (1996). Leitfaden für das Sprachenlernen im Tandem über das Internet (Manuskripte zur Sprachlehrforschung. 52). Bochum: Brockmeyer. Disponible en http://www.slf.ruhruni-bochum.de/learning/tanbib.html. [Consulta: 2011, 29 de noviembre].

Chi-Fen, E.C. (2006). The development of e-mail literacy: From writing to peers to writing to authority figures. Language Learning and Technology, 10(2), 35-55.

Crystal, D. (2001). Language and the Internet. Cambridge: Cambridge UP.

Danet, B. (2002). The language of email. Disponible en: http://www.europhd.eu/html/_onda02/04/ss8/pdf_files/lectures/Danet_email.pdf. [Consulta, 2011, 29 noviembre].

Gläsmann, S. \& Calvert, M. (2001). Tandem language learning in lchools. Sheffield: Philip Armstrong Publications.

Gonglewski, M., Meloni, Ch, \& Brant, J. (2001). Using e-mail in foreign language teaching: Rationale and suggestions. The Internet TESL Journal, VI(3). Disponible en: http://iteslj.org/Techniques/MeloniEmail.html. [Consulta: 2011, 29 de noviembre].

Greenbaum, S. \& Quirk, R. (1993). A student's grammar of the English language. Essex: Longman.

Jiménez Catalán, R.M \& J. Ojeda Alba, J. (2007a). The worlds children’s words build. Didáctica, 19, 155-172.

Jiménez Catalán, R.M. \& Ojeda Alba, J. (2007b). La carta como instrumento de identificación personal e interacción comunicativa en L2: Análisis del vocabulario de los saludos y despedidas. En J. Ramírez (coordinador). La lengua escrita (pp. 503-511). SEDLL/Universidad de La Rioja.

Jiménez Catalán, R.M. \& Mancebo Francisco, R. (2008). Vocabulary input in EFL textbooks. RESLA, 21, 147165. 
Jiménez Catalán, R.M. \& Ojeda Alba, J. (2008). The English vocabulary of girls and boys: Evidence from a quantitative study. En K. Harrington, L. Litosseliti, H. Sauntson y J. Sunderland (comps.), Gender and language research methodologies (pp. 103-115). Basingstoke: Palgrave Macmillan.

Little, D. (1998). Technologies, media and foreign language learning. Dublin: Authentik.

Nagel, P.S. (1999). E-mail in the virtual ESL/EFL classroom. The Internet TESL Journal, V(7). Disponible en: http://iteslj.org/Articles/Nagel-Email.html. [Consulta: 2011, 29 de noviembre].

Nation, P. S. N. (2001). Learning vocabulary in another language. Cambridge: Cambridge University Press.

Ojeda Alba, J. (2010). Themes and vocabulary in CLIL and non-CLIL instruction. En Y. Ruiz de Zarobe, \& R. M. Jiménez Catalán (comp.), Content and Language Integrated Learning. (pp.130-156).Bristol: Multilingual Matters.

Vinagre, M. (2005). Intercultural communication in action: A Project in tandem learning via e-mail. LinRed, 115.

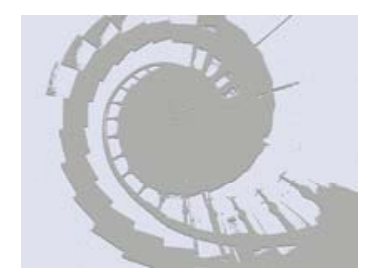

